\title{
A neuro-fuzzy approach to the reliable recognition of electric earthquake precursors
}

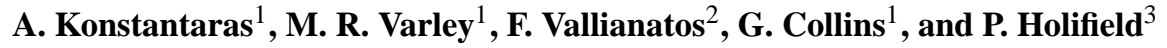 \\ ${ }^{1}$ University of Central Lancashire, ADSIP Research Centre, Department of Technology, Preston PR1 2HE, UK \\ ${ }^{2}$ Technological Educational Institute of Crete, Department of Natural Resources Engineering, Laboratory of Geophysics and \\ Seismology, Chania, Crete, Greece \\ ${ }^{3}$ University of Central Lancashire, Faculty of Design and Technology, Preston PR1 2HE, UK
}

Received: 1 July 2004 - Revised: / - Accepted: 11 October 2004 - Published: 18 October 2004

Part of Special Issue "Precursory phenomena, seismic hazard evaluation and seismo-tectonic electromagnetic effects"

\begin{abstract}
Electric Earthquake Precursor (EEP) recognition is essentially a problem of weak signal detection. An EEP signal, according to the theory of propagating cracks, is usually a very weak electric potential anomaly appearing on the Earth's electric field prior to an earthquake, often unobservable within the electric background, which is significantly stronger and embedded in noise. Furthermore, EEP signals vary in terms of duration and size making reliable recognition even more difficult. An average model for EEP signals has been identified based on a time function describing the evolution of the number of propagating cracks. This paper describes the use of neuro-fuzzy networks (Neural Networks with intrinsic fuzzy logic abilities) for the reliable recognition of EEP signals within the electric field. Pattern recognition is performed by the neural network to identify the average EEP model from within the electric field. Use of the neuro-fuzzy model enables classification of signals that are not exactly the same, but do approximate the average EEP model, as EEPs. On the other hand, signals that look like EEPs but do not approximate enough the average model are suppressed, preventing false classification. The effectiveness of the proposed network is demonstrated using electrotelluric data recorded in NW Greece.
\end{abstract}

\section{Introduction}

Prior to an earthquake, which is a result of the collision between two plates of the Earth, energy is stored in the seismogenic area. The release of a significant amount of this energy causes an earthquake. Some of this energy is triggered in the preparatory stage of the seismic process (Teisseyre, 1995). Release of this energy results in a number of micro-cracks, producing a weak electric signal, according to

Correspondence to: F. Vallianatos

(fvallian@chania.teicrete.gr) the theory of propagating cracks (Teisseyre and Nagahama, 1999; Vallianatos and Tzanis, 1998). Initially, there is a rapid rise in the number of propagating cracks, which creates a transient electric field. The whole process typically lasts in the order of several tens of minutes, and the resulting electric signal is considered as an Electric Earthquake Precursor (EEP) (Tzanis et al., 2000). Such signals may be observed on measurements of the electric field of the Earth, and are of great interest to the seismological society. However EEPs are relatively weak with respect to the electric background and their presence is not always clear (Colangelo at al., 2000). In addition, reliable recognition of EEPs is further complicated by noise (e.g. noise of magnetotelluric origin (Kaufman and Keller, 1981) caused mainly by magnetic storms, electrochemical noise due to change in the contact potential between electrodes and ground during and after rain, anthropogenic noise mainly due to power-lines in the vicinity of the observation station). More importantly though, reliable recognition of EEP signals is made difficult by their nature: EEPs vary considerably in terms of size and duration. Low magnitude seismic events often produce weaker EEP signals than those preceding a sizeable earthquake. In addition EEP signals vary in duration lasting from a few minutes to several tens of minutes without any pattern emerging relating them with the size of the forthcoming seismic event (Vallianatos et al., 2002).

This paper presents a method for the recovery of EEP signals based on an average EEP model and Adaptive NeuroFuzzy Inference Systems (ANFIS). There are a few different types of signals that are believed to be promising candidates for electric earthquake precursors (Tzanis and Vallianatos, 2001). Attempting to recognise all of them is inappropriate, due to the vague nature or lack of scientific description of most of them. The initial approach of this work is to deal with one particular type of EEP, which is based on a strong theoretical background, i.e. normalised time functions that 


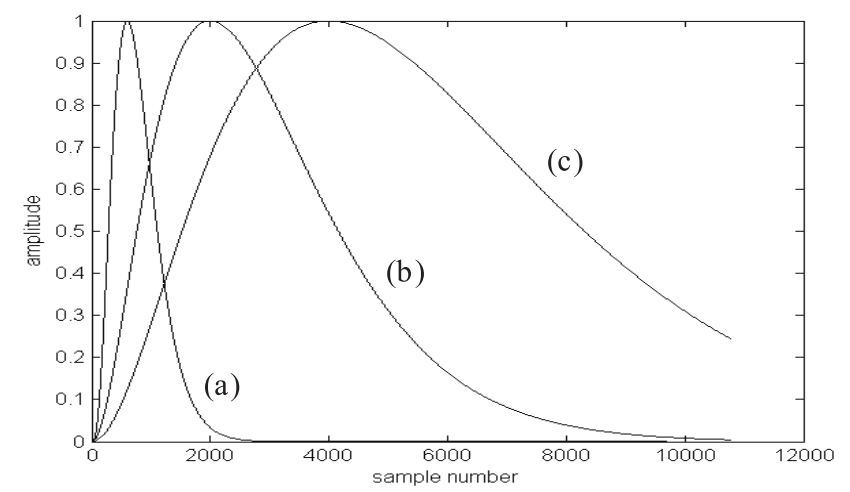

Fig. 1. Normalised simulated EEP signals.

describe the evolution of the number of propagating cracks. This type of EEP may be described by the following formula (Tzanis and Vallianatos, 2001):

$E(t)=(A t)^{\beta} e^{-\lambda t}$

in which $\beta$ controls the rate of rise of the signal whilst $\lambda$ controls the decay, and $A$ is a scale factor controlling the amplitude of the EEP.

Figure 1 shows three signals, each normalised to have a maximum amplitude of unity, that have been generated using this formula.

a) $A=0.2 \times 10^{-7} \quad \beta=3 \quad \lambda=5 \times 10^{-3}$

b) $A=0.3 \times 10^{-6} \quad \beta=2 \quad \lambda=1 \times 10^{-3}$

c) $A=0.2 \times 10^{-7} \quad \beta=2 \quad \lambda=5 \times 10^{-4}$

The EEP signals range in terms of duration from a few minutes to several tens of minutes and have significantly lower amplitude than the electric field. The average EEP model, resulting from statistical analysis of pre-observed EEP signals, coincides with signal (a) on Fig. 1 and has a duration of approximately 35 min whilst its amplitude is typically $2 \mathrm{~dB}$ less than the electric field.

\section{Neuro-fuzzy models}

Neuro-fuzzy models are neural networks with intrinsic fuzzy logic abilities, i.e. the weights of the neurons in the network define the premise and consequent parameters of a fuzzy inference system. Premise parameters determine the shape and size of the input membership functions, whilst consequent parameters determine the characteristics of the output membership functions and define the rules guiding the fuzzy inference system. The Adaptive Neuro-Fuzzy Inference System (ANFIS) algorithm (Jang et al., 1996) generates a fuzzy inference system which maps an input data set to an output data set by adjusting its membership functions using a hybrid algorithm: a combination of the error back-propagation algorithm and the least squares method. This requires less computation than the back-propagation algorithm alone, since the method of least squares reduces the search space dimensions of the original back-propagation algorithm. In a similar way to feed-forward neural networks, ANFIS generates an output signal during the forward pass, based on the initial set of membership functions. The consequent parameters are defined in the forward pass using the least squares method. Then the output is compared to the required output, and an error signal is fed back to the system to be used during the backward pass to alter the premise parameters using the back-propagation algorithm. This process continues for a number of epochs until the error signal becomes acceptably small (ideally zero). Figure 2 shows the ANFIS architecture of a two-input ( $x$ and $y$ ) neuro-fuzzy network guided by nine rules, where each input is assumed to have three associated membership functions:

Adaptive nodes $A_{1}, A_{2}, A_{3}$ and $B_{1}, B_{2}, B_{3}$ hold the parameters of the input membership functions, whilst adaptive nodes 1 to 9 hold the parameters of the output membership functions and the rules guiding the network. Nodes labelled as $\Pi$ are fixed and compute the firing strength of a rule, whilst nodes labelled as $\mathrm{N}$ compute the ratio of a rule's firing strength to the sum of all rules' firing strengths.

\subsection{Grid partitioning}

To generate an initial fuzzy inference system, grid partitioning (Jang et al., 1996) is applied to the input data of an input/output data set. In the case of the neuro-fuzzy model shown in Fig. 2, the two-dimensional input space is partitioned into nine overlapping fuzzy regions, each of which is governed by a fuzzy if-then rule, as shown in Fig. 3. The structure of the neuro-fuzzy model depends on the number of inputs and input membership functions per input. Each rule and membership function is represented by a single neuron. The rules guiding the fuzzy inference system are equal to the number of membership functions per input to the power of the total number of inputs of the system. A single neuron is used as bias to define the importance of either rule in the system. Each rule is linked to a single output membership function. A single neuron is used to produce a crisp output (defuzzification).

\section{Application of ANFIS for EEP recognition}

ANFIS has been shown to be effective as a universal approximator which can match any input/output data set, providing the system is adequately trained (Konstantaras, 2000). Here, the requirement is to use the noisy electric field signal, possibly including one or more EEPs, as input to the neuro-fuzzy model, which should then produce an estimate of the EEP(s) (Konstantaras et al., 2002). Training input data is provided in the form of noisy electric field meter readings including the simulated average EEP signal(s), with the corresponding simulated EEP(s) as the required output. Following training, the system's performance is tested using signals unseen during training. 


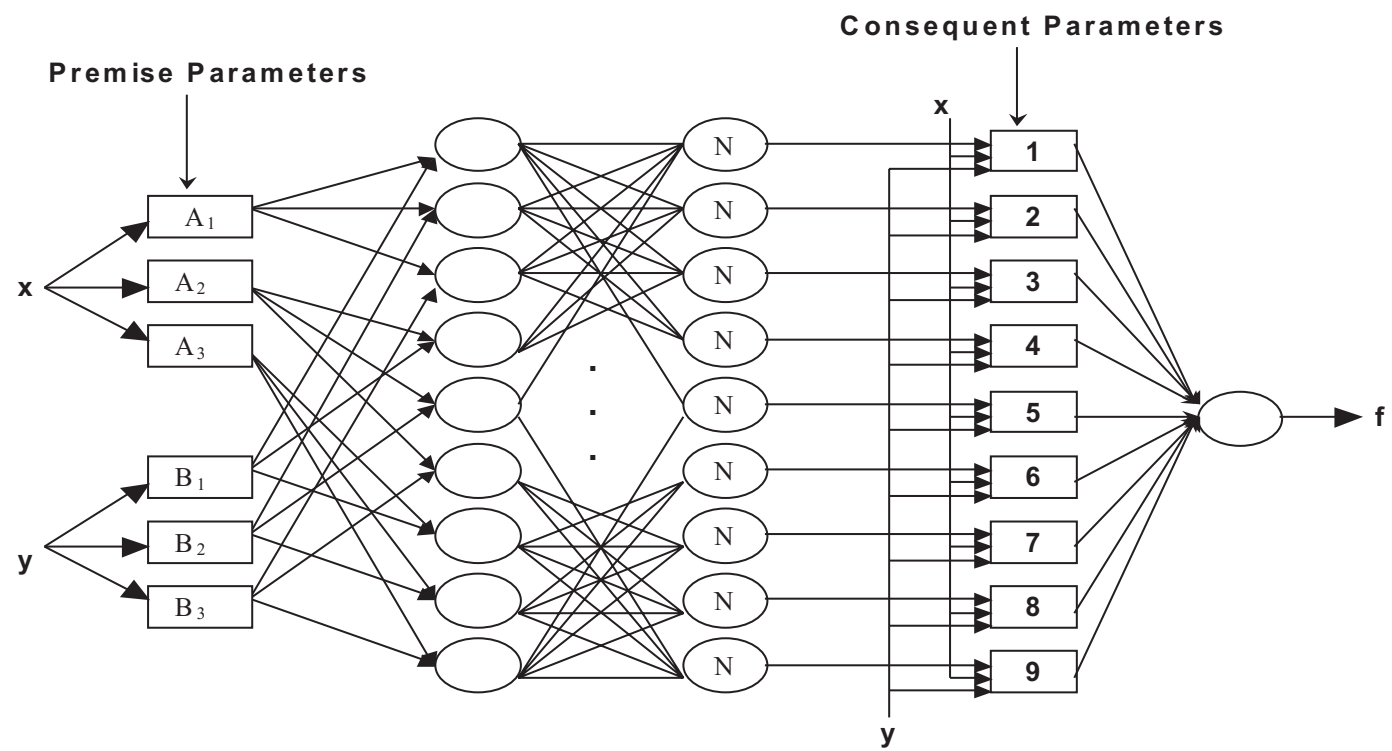

Fig. 2. ANFIS architecture.

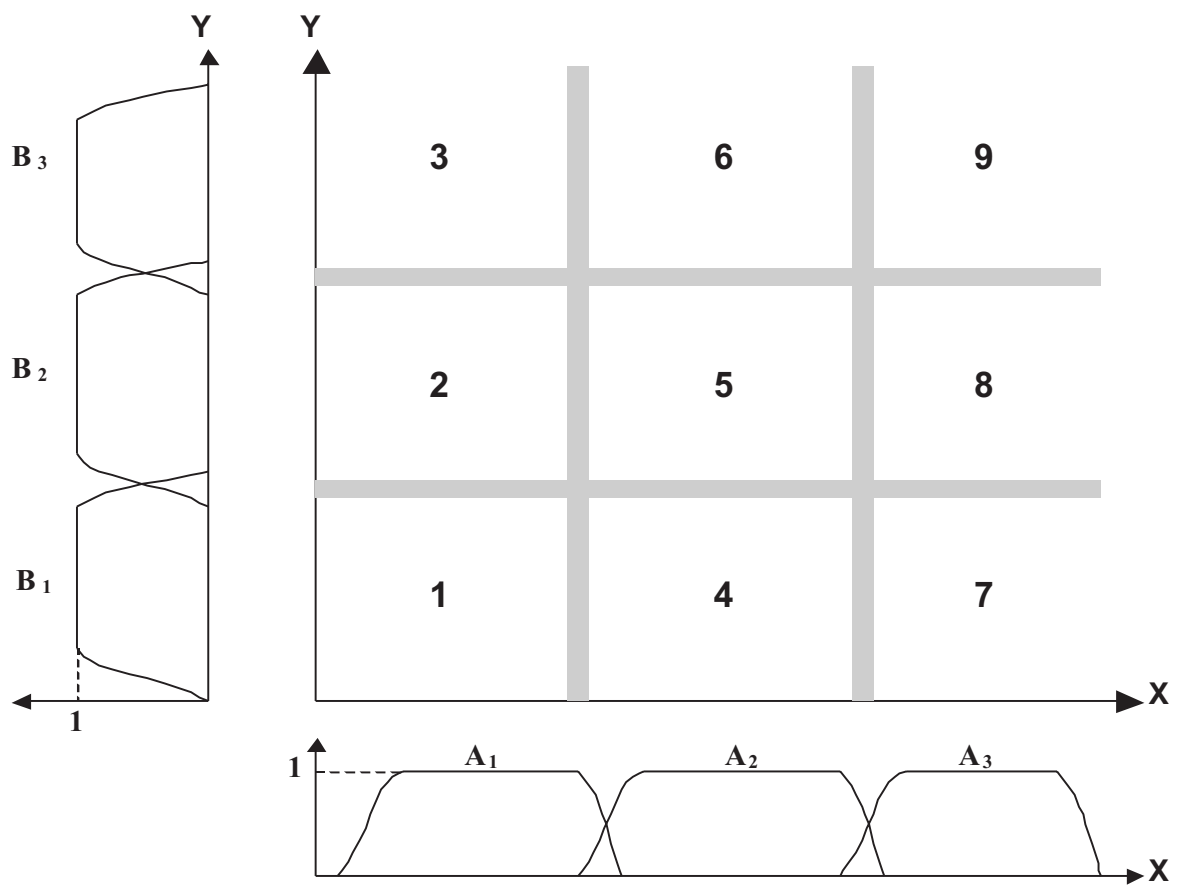

Fig. 3. Input space partitioning into nine fuzzy regions.

\subsection{Non-linear inverse adaptive modelling}

This method proposes a sliding window, containing the current input and a number of previous inputs, as input to the system. In this way ANFIS does not perform one-to-one mapping by simply matching each input to an output, but instead looks back in the input signal and retrieves information such as slopes, rising edges and falling edges, and information on their duration. This method capitalises on the neural network architecture of ANFIS which enables it to retrieve the different properties of the signals appearing at its input, i.e. in this case EEPs and electric background. Furthermore, this method is superior to pattern recognition techniques using neural networks alone, as the intrinsic fuzzy inference system enables the network to recognise EEP signals that are similar, but not identical, to the average EEP model. Signals that would otherwise be probable candidates for EEPs, but are not close enough to the chosen average model, are suppressed. 


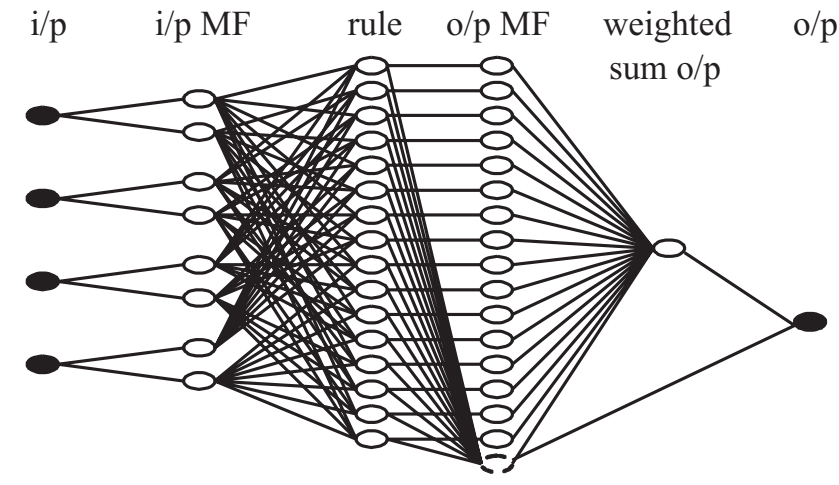

nodes - black: i/p - o/p, white: neurons, dashed: bias

Fig. 4. Neuro-fuzzy model structure.

\subsection{Neuro-fuzzy model architecture}

The structure of the neuro-fuzzy model produced by the grid partitioning of the input space and trained by ANFIS for this application is shown in Fig. 4. Each input (layer 1) has two input membership functions (layer 2), and hence there are $2^{4}=16$ rules (layer 3 ) and sixteen output membership functions (layer 4). The neuron on layer 5 sums the outputs of each rule weighted by the corresponding output membership function to produce a crisp output (layer 6).

\section{Layer 1}

The current sample and three previous samples are used as inputs (A, B, C, D) introducing recursion to the network.

\section{Layer 2}

Every node $i$ in this layer is an adaptive node with a node function:

$O_{1, i}=\mu A_{i}(x), \quad$ for $i=1,2$, or

$O_{1, i}=\mu B_{i-2}(y)$, for $i=3,4$, or

$O_{1, i}=\mu C_{i-4}(z)$, for $i=5,6$, or

$O_{1, i}=\mu D_{i-6}(k)$, for $i=7,8$

where $x$ (or $y$, or $z$, or $k$ ) is the input to node $i$ and $A_{i}$ (or $B_{i}$, or $C_{i}$, or $D_{i}$ ) is the equivalent membership function. The type of membership function $A$ (and $B, C, D$ ) is that of the generalised bell function:

$\mu A(x)=\frac{1}{1+\left|\frac{x-c_{i}}{a_{i}}\right|^{2 b}}$,

where $\left\{a_{i}, b_{i}, c_{i}\right\}$ are the premise parameters of the network determining the shape and size of the membership function. In a similar way to feed-forward neural networks, the premise parameters are adjusted during the backward pass of every training epoch by the error back-propagation algorithm.

\section{Layer 3}

Every node in this layer is a fixed node calculating the normalised firing strength of each rule, with a node function:

$O_{2, i}=\overline{w_{i}}=\frac{w_{i}}{\sum_{i} w_{i}}$,

where

$w_{i}=\mu A_{i}(x) \cdot \mu B_{i}(y) \cdot \mu C_{i}(z) \cdot \mu D_{i}(k)$

\section{Layer 4}

Every node $i$ in this layer is an adaptive node using the output membership functions to compute the weighted output of each rule, according to the following node function:

$O_{3, i}=\overline{w_{i}} f_{i}=\overline{w_{i}}\left(p_{i} x+q_{i} y+m_{i} z+n_{i} k+r_{i}\right)$,

where $\left\{p_{i}, q_{i}, m_{i}, n_{i}, r_{i}\right\}$ are the consequent parameters of the network defining the rules of the fuzzy inference system. The least squares estimator adjusts the consequent parameters during the forward pass of every training epoch. The dashed node in Layer 4 is a bias input, the weights of which are used to define the importance of each rule in the system.

\section{Layer 5}

The single node in this layer is a fixed node, which converts the weighted fuzzy outputs of the rules in the system rule into a single crisp output, as described by the following node function:

$O_{4,1}=\sum_{i} \bar{w}_{i} f_{i}$

\section{Layer 6}

The actual output of the neuro-fuzzy model for a given set of input data.

\subsection{ANFIS training and EEP recognition}

To produce the input signal the time domain electric field signal was measured, and simulated average EEP signals were added on. Inverse adaptive modelling was used, i.e. the electric background including EEPs was presented as input to the ANFIS system, and the EEPs alone as the required output. All the recordings of the electric field were taken at a sampling frequency of $1 \mathrm{~Hz}$. Since EEPs are low frequency signals in comparison to the electric field, the signals were decimated and down-sampled by a factor of eight. The continuous line in Fig. 5 shows the processed electric signal, whilst the dotted line indicates the exact location of the EEP signals.

The first 200 samples of the signal shown in Fig. 5 are stored as bank-data to enable initialisation of the training process of the neuro-fuzzy model. Large amounts of bank-data 


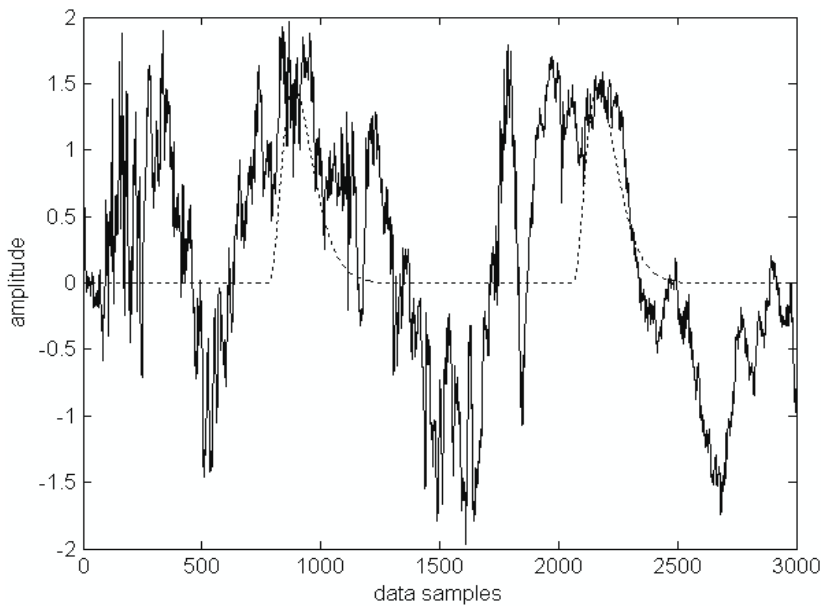

Fig. 5. Decimated and downsampled real electric time-series (continuous line) carrying two EEP signals indicated by the dotted line.

can result in a slow start for the network, as the system is not fully operational until an equal amount of data samples has been processed. From the remaining 2800 data points, the first 1400 samples are used for training purposes whilst the following 1400 data points remain unseen by the neuro-fuzzy model and are used to evaluate its performance.

The neuro-fuzzy model has four inputs, one for the current input data $(n)$ whilst the remaining three inputs hold earlier data samples spread apart by fifty places $(n-50, n-100$ and $n-150)$. The required output is that of the EEP signals only. The wide spread between the four inputs enables the neurofuzzy model to detect low frequency rising and falling slopes which are more likely to belong to an EEP, and to ignore high frequency edges caused mainly by the electric field.

The training process of the neuro-fuzzy model is a result of a hybrid algorithm, a combination of the least squares estimator and the error back-propagation algorithm. In the forward pass it computes a crisp output based on the input signals, the weighted rules' outputs and the shape of the input and output membership functions. The least squares estimator adjusts the consequent parameters of the fuzzy inference system. The difference between the actual and required output is calculated and used as an error signal. During the backward pass of the hybrid algorithm (error back-propagation), the error value of the last training epoch is used to adapt the premise parameters of the fuzzy inference system. This process carries on for as many training epochs as necessary to reduce the error signal to an acceptable level (ideally zero).

This process is inherently adaptive, because of the adaptive nature of the neural networks within ANFIS. This is demonstrated by training ANFIS to classify EEPs for a specific signal and then applying the resulting model to further recordings of the electric field unseen by the neuro-fuzzy model. The successfully trained model compensates for the dynamic nature of the electric field and for any distortions appearing on the electrotelluric recordings of a given observation station.

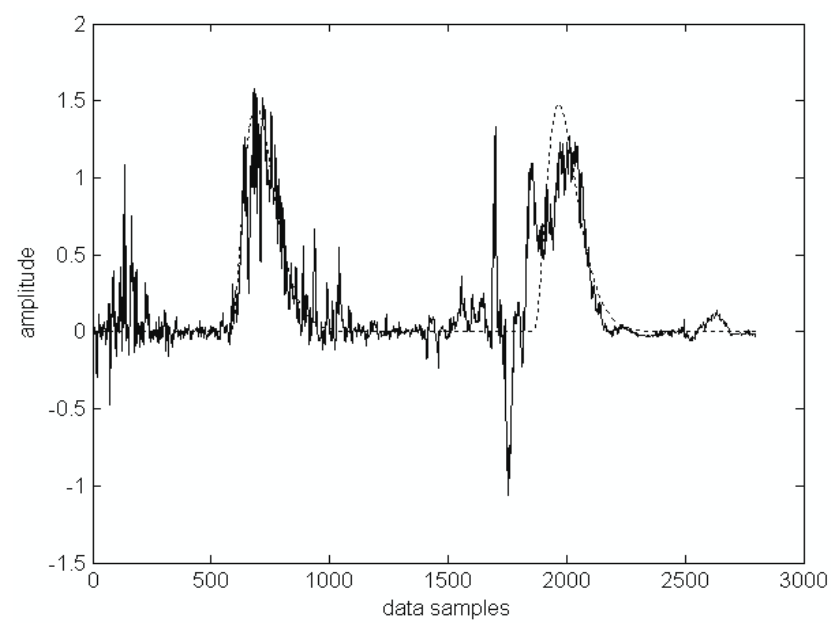

Fig. 6. ANFIS output for seen and unseen signals, the System's output is given by the continuous line, while the EEP signals is the dotted line.

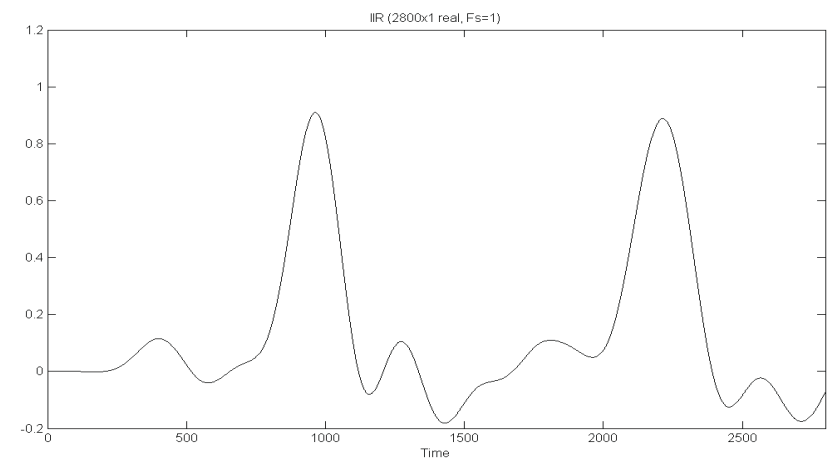

Fig. 7. Post-processed ANFIS output.

\section{Experimental results}

The proposed neuro-fuzzy model was tested by applying it on electrotelluric data (Fig. 5) collected at the area of Ioannina in North-West Greece. ANFIS was trained using the first half of the recorded electric field signal including a simulated EEP, with the simulated EEP signal alone as the required output. The trained system was then tested using the unseen part of the electric field signal, with an additional simulated EEP of known location and duration included.

Figure 6 demonstrates the performance of the neuro-fuzzy model for a previously seen signal, i.e. training data, and an unseen signal, plotted next to each other. The required output (dotted line) is plotted against the output of the neuro-fuzzy model (continuous line). The first 1400 data points demonstrate the performance of the neuro-fuzzy model for the seen signal, whilst the following 1400 data points demonstrate the performance of the model for the unseen signal.

It is clear that the system output closely approximates the required output, thereby successfully detecting the EEP signals and clearing the background noise caused by the Earth's electric field. To ensure reliable recognition of the EEP 
signals the output signal is post-processed with a 6th order IIR low-pass filter with a cut-off frequency of $2 \mathrm{mHz}$, removing the unwanted high frequency component and providing a clear indication of the EEPs, as shown in Fig. 7.

\section{Concluding remarks}

In this paper, a neuro-fuzzy ANFIS system is presented, which has been successfully trained to recognise electric earthquake precursors appearing on electric field recordings. A simulated average EEP model has been developed and used for training the system. Testing with unseen data verified the effectiveness of the model.

This method is superior to stand-alone neural networks (Rovithakis and Vallianatos, 2000), as the incorporation of fuzzy logic in the system enables it to recognise EEPs that approximate, but are not identical to, the average EEP model, whilst suppressing non-EEP signals which may otherwise be falsely classified as EEPs.

Furthermore, comparing this method with conventional methods, e.g. residual electric field calculations (Hadjioannou et al., 1993), an advantage of the neuro-fuzzy technique is that any disturbances induced to the electrotelluric recordings are treated as part of the electric field and do not effect the reliable recognition of electric earthquake precursors.

Future work will focus on the development of an intrinsic real-time on-line learning algorithm for neuro-fuzzy models. The dynamic nature of the electric field and the wide variation amongst EEP signals indicate that it is highly unlikely for a fixed set of electrotelluric data to contain all the main features of the related signals. A neuro-fuzzy model continuously adapting its parameters during operation would eventually be able to identify all the main characteristics of the related signals, compensate for the dynamic nature of the background environment and recognise a range of EEP signals despite variations.

Edited by: P. F. Biagi

Reviewed by: V. Lapenna and another referee

\section{References}

Colangelo, G., Lapenna, V., Vallianatos, F., and Nomikos, C.: Investigating the time dynamics of geoelectrical signals measured in two seismotectonic environments of Mediterranean region: the Southern Apennine chain (S. Italy) and the Hellenic Arc (Crete island, Greece), Annali di Geofisica, 43/2, 391-408, 2000.

Hadjioannou, D., Vallianatos, F., Eftaxias, K., Hadjicontis, V., and Nomikos, K.: Subtraction of the telluric inductive component from VAN measurements, Tectonophysics, 224 (1), 113-124, 1993.

Jang, J.-S. R., Sun, C.-T., and Mizutani, E.: Neuro-Fuzzy and Soft Computing, Prentice Hall, 1996.

Kaufman, A. A. and Keller, G. V.: The Magnetotelluric Sounding Method, Elsevier, 1981.

Konstantaras, A: Fuzzy-Neural Methods for Modelling and Control, MSc thesis, University of Portsmouth, 2000.

Konstantaras, A., Varley, M. R., Vallianatos, F., Collins, G., and Holifield, P.: Recognition of Electric Earthquake Precursors using Neuro-Fuzzy models: Methodology and Simulation Results, Signal Processing Pattern Recognition and Analysis, IASTED, Crete, Greece, 2002.

Rovithakis, G. A. and Vallianatos, F.: A neural network approach to the identification of Electric Earthquake Precursors, Phys. Chem. Earth, Vol. 25, No. 3, 315-319, 2000.

Teisseyre, R. (Ed): Theory of Earthquake premonitory and fracture processes, Polish Scientific Publishers, 1995.

Teisseyre, R. and Nagahama, H.: Dislocational models of electric field generation in a seismic source zone, in: Atmospheric and Ionospheric Electromagnetic Phenomena Associated with Earthquakes, edited by: Hayakawa, M., Terra Scientific Publishing, Tokyo, 271-285, 1999.

Tzanis, A., Vallianatos, F., and Gruszow, S.: Identification and discrimination of transient electrical earthquake precursors: Fact, fiction and some possibilities, Phys. Earth. Planet. Int., 121, 223 248, 2000.

Tzanis, A. and Vallianatos, F.: A critical review of Electric Earthquake Precursors, Annali di Geofisica, 44/2, 429-460, 2001.

Vallianatos, F. and Tzanis, A.: Electric current generation associated with the deformation rate of a solid: Preseismic and coseismic signals, Phys. Chem. Earth, 23, 933-938, 1998.

Vallianatos, F., Lapenna, V., Troyian, V., Smirnova, N., Kopytenko, Y., Korepanov, V., and Matiashvili, T.: Study of the ULF electromagnetic phenomena related to earthquakes: Strategy of the SUPRE project, Seismo Electromagnetics (LithosphereAtmosphere-Ionosphere Coupling), Terrapub, 2002. 